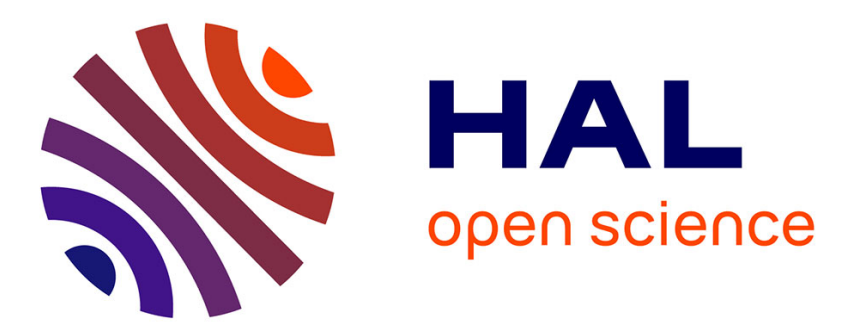

\title{
FLUCTUATIONS D'INTENSITÉ ACOUSTIQUE D'UNE ONDE SPHÉRIQUE TRAVERSANT UNE TURBULENCE THERMIQUE
}

P. Blanc-Benon, D. Juve

\section{- To cite this version:}

P. Blanc-Benon, D. Juve. FLUCTUATIONS D'INTENSité ACOUSTIQUE D'UNE ONDE SPHÉRIQUE TRAVERSANT UNE TURBULENCE THERMIQUE. Journal de Physique Colloques, 1990, 51 (C3), pp.C3-13-C3-22. 10.1051/jphyscol:1990302 • jpa-00230730

HAL Id: jpa-00230730

https://hal.science/jpa-00230730

Submitted on 1 Jan 1990

HAL is a multi-disciplinary open access archive for the deposit and dissemination of scientific research documents, whether they are published or not. The documents may come from teaching and research institutions in France or abroad, or from public or private research centers.
L'archive ouverte pluridisciplinaire HAL, est destinée au dépôt et à la diffusion de documents scientifiques de niveau recherche, publiés ou non, émanant des établissements d'enseignement et de recherche français ou étrangers, des laboratoires publics ou privés. 


\title{
FLUCTUATIONS D'INTENSITÉ ACOUSTIQUE D'UNE ONDE SPHÉRIQUE TRAVERSANT UNE TURBULENCE THERMIQUE
}

\author{
P. BLANC-BENON et D. JUVE \\ Laboratoire de Mécanique des Fluides et d'Acoustique, CNRS URA-263, \\ Ecole Centrale de Lyon, BP 163, F-69131 Ecully Cedex, France
}

Résumé : L'étude expérimentale présentée permet de caractériser les fluctuations d'intensité acoustique d'une onde sphérique traversant une furbulence thermique. Comple tenu des distances de propagation importantes nous mettons en évidence une saturation des fluctuations d'intensité. La loi de distribution de ces fluctuations est caractérisée depuis les faibles fluctuations jusqu'au fortes fluctuations. Nous comparons nos mesures avec les estimations déduites de différentes densités de probabilité (log-normale, exponentielle, 1-K, gamma généralisée).

Abstract : The intensity fluctuations of a spherical wave that propagates through a turbulent thermal field are investigated. Results are given for the normalized variance and the probabilitydensity function of intensity fluctuations under a variety of propagation conditions. Measured probability densities are compared with the log-normal, the exponential, the I- $\mathrm{K}$ and the generalized gamma distributions.

\section{1 - INTRODUCTION}

L'analyse des phénomènes de propagation des ondes dans les milieux aléatoires est à l'origine de très nombreux travaux tant théoriques qu'expérimentaux, avec des applications en acoustique aérienne ou sous-marine pour les problèmes d'imagerie, en optique pour la propagation des faisceaux laser dans l'atmosphère. Compte tenu du caractère aléatoire du milieu, une onde en se propageant subit des modifications de ses caractéristiques spatio-temporelles. On observe alors des fluctuations de phase et d'amplitude qui se traduișent notamment par des atténuations et des fluctuations d'intensité importantes pour l'onde transmise.

Les problèmes liés à l'intensité moyenne de l'onde transmise et à sa perte de cohérence spatiale peuvent être résolus de façon satisfaisante à partir du modèle introduit par Tatarski /1/. En effet, le moment d'ordre deux en deux points du champ transmis est solution d'une équation parabolique déduite de l'équation d'Helmholtz à indice aléatoire. Une fois précisé le type d'onde incidente (plane, sphérique, faisceau gaussien), on calcule une solution pour le moment d'ordre deux en prenant en compte les différentes échelles de furbulence mises en jeu grâce à une modélisation appropriée du spectre tridimensionnel des fluctuations d'indice (Tatarski /1/, Ishimaru 12/).

Pour les fluctuations d'intensité acoustique, qui sont reliées au moment d'ordre quatre du champ transmis, les problèmes de la prédiction de leur niveau et surtout de leur loi de probabilité ne sont pas entièrement résolus. Pour les faibles niveaux de fluctuations la solution de Rytov (Tatarski /1/) permet de prédire les variations

du logarithme de l'amplitude de l'onde transmise $\chi=\operatorname{Ln} A$. Les fluctuations d'amplitude sont alors réparties suivant une loi log-normale. Pour les très fortes fluctuations on observe une saturation des fluctuations d'intensité qui sont alors distribuées suivant une loi exponentielle (Prokhorov et al./3/, Strohbehn/4/, Uscinski /5/, Gracheva et al. /6/, Furutsu /7//.

Nous présentons ici des résultats expérimentaux concernant la variance des fluctuations d'intensité $\sigma_{I}^{2}$ et les densités de probabilité de ces fluctuations, pour une onde sphérique traversant une turbulence thermique. Cette élude complémentaire des travaux antérieurs (Blanc-Benon, Juvé /8/) couvre un très large domaine s'étendant depuis le régime des faibles fluctuations jusqu'à celui de la saturation. Pour ce qui est de la variance 
$\sigma_{\mathrm{I}}^{2}$ nous comparons nos mesures aux différentes estimations théoriques disponibles : solution de Rytov, solutions asymptotiques de l'équation d'évolution du moment d'ordre quatre. Les densités de probabilités expérimentales sont comparées aux distributions classiques (log. normale et exponentielle) ainsi qu'à des lois à deux paramètres (loi gamma généralisée (Blanc-Benon /9/), loi I-K (Andrew, Philipps, Shivamozzi /10/) qui permettent de décrire la zone intermédiaire des fluctuations "moyennes" dans laquelle aucune évaluation théorique n'est disponible.

\section{2 - INSTALLATION EXPERIMENTALE}

Pour que les mesures effectuées soient représentatives des essais de propagation dans la turbulence réalisés "in situ" dans l'atmosphère ou dans l'océan il est nécessaire de vérifier certaines conditions. En particulier la distance de propagation dans la turbulence $x$ doit être grande devant l'échelle de corrélation spatiale $L_{T}$ des fluctuations de température, et la longueur d'onde acoustique $\lambda$ doit être petite vis-à-vis de celte même échelle $\mathrm{L}_{\mathrm{T}}$. Dans linstallation réalisée en chambre anéchoïde $(10 \mathrm{~m} \times 8 \mathrm{~m} \times 7 \mathrm{~m})$ le volume de turbulence est créé par mélange de panaches thermiques développés à partir de résistances électriques disposées sous forme de grille (Fig. 1). L'ensemble, constitué de deux grilles identiques de dimension $110 \mathrm{~cm} \times 220 \mathrm{~cm}$ avec une maille $\mathrm{M}$ de $9 \mathrm{~cm}$ et une puissance électrique de $32 \mathrm{~kW}$, fonctionne en convection libre. Lorsque les deux grilles sont mises bout à bout la distance maximale de propagation dans la turbulence est de $440 \mathrm{~cm}$, la valeur efficace des fluctuations de température est de $5^{\circ} \mathrm{C}$, d'où un taux $\sqrt{\mathrm{T}^{2}} / \overline{\mathrm{T}}$ de $1,710^{-2}$, et l'échelle de corrélation spatiale $\mathrm{L}_{\mathrm{T}}$ est de $7.6 \mathrm{~cm}$. Lorsque les deux grilles sont superposées et décalées de façon à réduire la maille $M$ de moitié, la distance de propagation maximale est de $220 \mathrm{~cm}$. Les fluctuations de température sont alors de $8,5^{\circ} \mathrm{C}$ d'où un taux $\sqrt{\mathrm{T}^{2}} / \overline{\mathrm{T}}$ de $2.510^{-2}$, et l'échelle intégrale $\mathrm{L}_{\mathrm{T}}$ est de $5 \mathrm{~cm}$ (Blanc-Benon $/ 9 /$ ).

Les sources acoustiques sont des émetteurs ultrasonores dont le diagramme de rayonnement utile est quasisphérique. Les fréquences d'émission f utilisées sont $23.5 \mathrm{kHz}, 31 \mathrm{kHz}, 39 \mathrm{kHz}$ et $75 \mathrm{kHz}$. Les signaux transmis sont reçus sur un ensemble de microphones Bruël \& Kjaer 1/4" disposés suivant l'axe propagation $x$. L'ensemble émetteur-récepteurs est situé dans un plan horizontal à une hauteur de $180 \mathrm{~cm}$ du plan des grilles dans une zone où l'homogénéité spatiale du champ de température est bonne. Les signaux de pression sont hétérodynés autour d'une fréquence de $5 \mathrm{kHz}$ et enregistrés sur magnétophone (Racal $14 \mathrm{DS}$ ). Pour obtenir les fluctuations d'intensité de l'onde transmise, hous utilisons un traitement numérique. Dans une première étape, les signaux sont digitalisés sur 12 bits avec $64 \mathrm{k} /$ mots de mémoire par voie (Difa Ts 9000) puis transférés sur une station de travail HP 9000/330 pour le stockage de masse. Dans une deuxième étape, à partir du signal de pression $\mathrm{p}$ numérisé on calcule les moments centrés de $\mathrm{I}$ soit $\left\langle(\mathrm{I}-\langle\mathrm{I}\rangle)^{\mathrm{n}}\right\rangle$ ainsi que les densités de probabilités des fluctuations d'intensité I. La fréquence d'échantillonnage est de $20 \mu \mathrm{s}$ ce qui donne une durée de $1.312 \mathrm{~s}$ par bloc de 65536 points. Les moyennes sont eflectuées sur 40 à $50 \mathrm{~s}$ de signal.

\section{3 - YARIANCE NORMALISEE DES FLUCTUATIONS D'INTENSITE}

Si I $(x, \vec{\rho})$ est l'intensité acoustique en un point $(x, \vec{\rho})$ d'un plan perpendiculaire à la direction de propagation $x$, la variance normalisée des fluctuations d'intensité sur l'axe $\sigma_{I}^{2}$ (appelée indice de scintillation en optique) est définie par :

$$
\sigma_{\mathrm{I}}^{2}=\frac{\left\langle\left(\mathrm{I}(\mathrm{x}, 0)-\langle\mathrm{I}(\mathrm{x}, 0)>)^{2}\right\rangle\right.}{\langle\mathrm{I}(\mathrm{x}, 0)\rangle^{2}}
$$

Pour les faibles niveaux de fluctuations, la solution de Rylov, qui est une méthode de perturbations "douces" appliquée à l'équation gouvernant l'évolution de l'iconale $\psi(x, \vec{\rho})=\operatorname{Ln}(p(x, \vec{\rho}))$, permet d'évaluer la variance $\sigma_{\chi}^{2}$ des fluctuations du logarithme de l'amplitude $\chi=\operatorname{Ln} A$ (Tatarski /1/). Dans le cas où l'onde incidente est 
sphérique on montre que (Ishimaru /2/) :

$$
\sigma_{x}^{2}=4 \pi^{2} k_{0}^{2} \int_{0}^{x} d z \int_{0}^{\infty} \sin ^{2}\left(\frac{x(x-z) K}{2 z k_{0}}\right) \cdot \phi_{E}(K) \cdot K \cdot d K
$$

où $\mathrm{k}_{\mathrm{o}}$ est le nombre d'onde acoustique ef $\phi_{\varepsilon}(\mathrm{K})$ est le spectre tridimensionnel des fluctuations $\varepsilon(x, \vec{\rho})=T^{\prime}(x, \vec{\rho}) / 2 \bar{T}(x, \vec{\rho})$. En modélisant $\phi_{\varepsilon}(K)$ par un spectre de Kolmogorov, qui ne prend en compte que la zone inertielle de turbulence, on obtient :

$$
\begin{aligned}
& \sigma_{\chi}^{2}=0.124 c_{\varepsilon}^{2} k_{o}^{\frac{7}{6}} x^{\frac{11}{6}} \\
& \phi_{\varepsilon}(K)=0,033 c_{\varepsilon}^{2} K^{-\frac{11}{3}} \quad c_{\varepsilon}^{2}=1.91\left(\frac{\sqrt{\overline{T^{2}}}}{2 \pi}\right)^{2} L_{o}^{-2 / 3}
\end{aligned}
$$

où $\mathrm{L}_{\mathrm{o}}$ est l'échelle externe de turbulence $\left(\mathrm{L}_{\mathrm{o}}=1.339 \mathrm{~L}_{\mathrm{T}}\right)$. Dans cette solution l'amplitude $A$ et l'intensité $\mathrm{I}$ sont distribuées suivant une loi log-normale et $\sigma_{1}^{2}$ se met sous la forme :

$$
\sigma_{I}^{2}=\exp \left(4 \sigma_{\chi}^{2}\right)-1
$$

La quantité $4 \quad \sigma_{\chi}^{2}$ a pour expression $0.5 c_{\varepsilon}^{2} \mathrm{k}_{0}^{7 / 6} \mathrm{x}^{11 / 6}$. II convient de noter que dans la littérature le paramètre le plus couramment utilisé est $\beta_{0}^{2}=1.23 c_{\varepsilon}^{2} k_{o}^{7 / 6} x^{11 / 6}$, qui correspond à la solution de Rytov pour une onde plane.

Dans le domaine des fortes fluctuations, l'approximation parabolique permel d'obtenir une équation régissant l'évolution du moment d'ordre quatre du champ de pression acoustique transmis (Tatarski /1/, Prokhorov et al, /3/, Uscinski et al. /11/). Pour une onde sphérique, en utilisant un spectre de Kolmogorov pour $\phi_{\varepsilon}(K)$, Gochelashvily et al. /12/ arrivent à l'expression asymptotique suivante :

$$
\sigma_{I}^{2} \approx 1+2,8\left(\beta_{0}^{2}\right)^{-2 / 5}\left(\sigma_{I}>1\right)
$$

Dans un travail récent, Andrew et al. $/ 10 /$, en supposant a priori que les flucluations d'intensité sont distribuées suivant une loi l-K à deux paramètres $\alpha$ ef $\rho$, montrent que $\sigma_{I}^{2}$ peut se mettre sous la forme :

$$
\begin{aligned}
& \sigma_{I}^{2}=\frac{2}{(1+\rho)^{2}}\left(\frac{1}{2}+\frac{1+\rho}{\alpha}\right) \\
& \alpha=0.71 \beta_{0}^{4 / 5} ; \rho=\frac{4.88}{\alpha \beta_{0}^{2}\left(1+0.2 \beta_{0}^{2}\right)}
\end{aligned}
$$

Les paramètres $\alpha$ et p sont estimés à partir des expressions de la variance pour les cas limites des faibles et des très fortes flucluations. 
Sur la figure 2, nous avons reporté en fonction de $\beta_{0}$ les valeurs de $\sigma_{I}^{2}$ mesurées en faisant varier la fréquence d'émission ( $\mathrm{f}=23.5 \mathrm{kHz}, 31 \mathrm{kHz}, 39 \mathrm{kHz}, 75 \mathrm{kHz}$ ), la distance de propagation dans la turbulence $(0.5 \mathrm{~m} \leq \mathrm{x} \leq 4.4 \mathrm{~m})$ et les caractéristiques de la turbulence $\left(\mathrm{L}_{\mathrm{T}}=5 \mathrm{~cm}, \sqrt{\mathrm{T}^{2}} / \overline{\mathrm{T}}=2.510^{-2}, \mathrm{~L}_{\mathrm{T}}=\right.$ $7.6 \mathrm{~cm}, \sqrt{\mathrm{T}^{2}} / \overline{\mathrm{T}}=1.710^{-2}$ ). On observe un bon regroupement des points en fonction du paramètre $\beta_{\mathrm{o}}$ avec toutefois des écarts sensibles dans la zone correspondant aux maixmum des fluctuations ; ces écarts sont vraisemblablement dus à une influence résiduelle du rapport de la longueur d'onde acoustique à l'échelle de turbulence. Pour les faibles valeurs l'accord avec la solution de Rytov est très satisfaisant. On note ensuite une croissance de $\sigma_{I}^{2}$ jusqu'à une valeur voisine de 2, puis une légère décroissance vers un seuil de saturation. Ces résultats sont en accord avec les mesures effectuées en optique par Gracheva et al. /6/ et Parry /13/. Les formules (4) et (5) permettent de prévoir l'allure générale de $\sigma_{I}^{2}$ en fonction de $\beta_{0}$.

Afin de caractériser de façon plus précise les différents régimes de propagation nous avons reporté l'ensemble des points expérimentaux sur un diagramme (Fig. 3) utilisant les paramètres $\Lambda$ et $\phi$ introduits par Flatté et al. /14/. Pour un spectre inertiel $\phi_{\varepsilon}(K) \approx K^{-11 / 3}$, ces paramètres s'expriment par : $\Lambda=x / k_{0} L_{T}^{2} \quad$ et $\phi^{2}=2 \mathrm{~L}_{\mathrm{T}} \mathrm{k}_{0}^{2} \times\left(\sqrt{\mathrm{T}^{2}} / 2 \widetilde{\mathrm{T}}\right)^{2}$. En traçant les courbes $\phi=1, \quad \Lambda \phi^{1,2}=1$ et $\Lambda \phi^{2,4}=1$ on détermine trois régions : la zone de saturation $\left(\Lambda \phi^{1,2}>1\right)$, la zone de validité de la solution de Rytov $\left(\Lambda \phi^{2,4}<1\right)$, et une zone intermédiaire où les fluctuations d'intensité sont dites partiellement saturées. On remarque que les points expérimentaux se répartissent dans ces trois régions, ce qui permet une description de caractéristiques des fluctuations d'intensité dans chaque régime. A titre d'illustration, nous avons tracé sur la figure 4 l'allure des fluctuations d'intensité I en fonction du temps pour des acquisitions simultanées à différentes distances de propagation correspondant à chacun des régimes de fluctuations. Dans la zone non saturée, la valeur moyenne de l'intensité est importante et les fluctuations se répartissent plus ou moins symétriquement autour de cette moyenne. Lorsqu'on se rapproche de la zone de saturation, on note l'apparition de pics très importants ainsi que de nombreux passages où le signal s'éteint pratiquement.

\section{4 - DENSITES DE PROBABILITES DES FLUCTUATIONS D'INTENSITE}

Dans le cas des faibles niveaux de fluctuations $\left(\beta_{0} \ll 1\right)$, correspondant au domaine de validité de la solution de Rytov, l'intensité est distribuée suivant une loi log-normale (Tatarski /1/) :

$$
W(I)=\frac{1}{\sqrt{2 \pi} \cdot I \cdot \sigma_{I}} \exp \left(-\left(\operatorname{Ln}\left(\frac{I}{\langle\mathrm{I}>}\right)+\frac{\sigma_{I}^{2}}{2}\right)^{2} \cdot \frac{1}{2 \sigma_{I}^{2}}\right)
$$

où l'écart-type $\sigma_{1}$ et la moyenne <l> sont estimés à partir des histogrammes expérimentaux. Pour le régime des très fortes fluctuations ( $\beta_{0}>1$ ) qui correspond à la zone de saturation, la loi généralement proposée pour les fluctuations d'amplitude est une distribution de Rayleigh d'où une loi exponentielle pour l'intensité (Strohbehn et al. /15/) :

$$
W(I)=\frac{1}{\langle\mathrm{I}\rangle} \exp \left(-\frac{\mathrm{I}}{<\mathrm{I}\rangle}\right)
$$

Pour les cas intermédiaires $\left(\beta_{0} \approx 1\right)$ il n'existe à notre connaissance aucune distribution établie à partir d'une analyse théorique rigoureuse. Certains auteurs proposent des termes correctifs à la statistique de 
Rayleigh (Dashen /16/) où la K-distribution (Parry /13/). En pratique ces lois ne semblent pas particulièrement adaptées (Ewart et al. 117/). Nous proposons d'utiliser la loi gamma généralisée à deux paramètres $b$ et $k$ soit :

$$
\begin{aligned}
& W(I)=b \mu^{k} / \Gamma(k) I^{b k-1} e^{-\mu I^{b}} \\
& \mu=\left(\frac{\Gamma(k+1 / b)}{\Gamma(k)}\right)^{b}
\end{aligned}
$$

où $\Gamma(k)$ est la fonction gamma. Lorsque $k \rightarrow \infty$ on retrouve la loi log-normale et lorsque $b=k=1$ on obtient la loi exponentielle. Pour évaluer les paramètres $b$ et $k$ nous résolvons le système non linéaire associé aux équations des moments d'ordre 2 et $3, \mathrm{~m}_{2}$ et $\mathrm{m}_{3}$ :

$$
\mathrm{m}_{2}=\frac{<\mathrm{I}^{2}>}{\left\langle\mathrm{I}{ }^{2}\right.}=\frac{\Gamma(\mathrm{k}) \Gamma(\mathrm{k}+2 / \mathrm{b})}{\Gamma^{2}(\mathrm{k}+1 / \mathrm{b})} \quad \mathrm{m}_{3}=\frac{<\mathrm{I}^{3}>}{\mathrm{II}^{3}}=\frac{\Gamma^{2}(\mathrm{k}) \Gamma(\mathrm{k}+3 / \mathrm{b})}{\Gamma^{3}(\mathrm{k}+1 / \mathrm{b})}
$$

Nous avons également effectué des comparaisons avec la loi de distribution proposée par Andrew et al. /10/, qui est une généralisation de la K-distribution, soil :

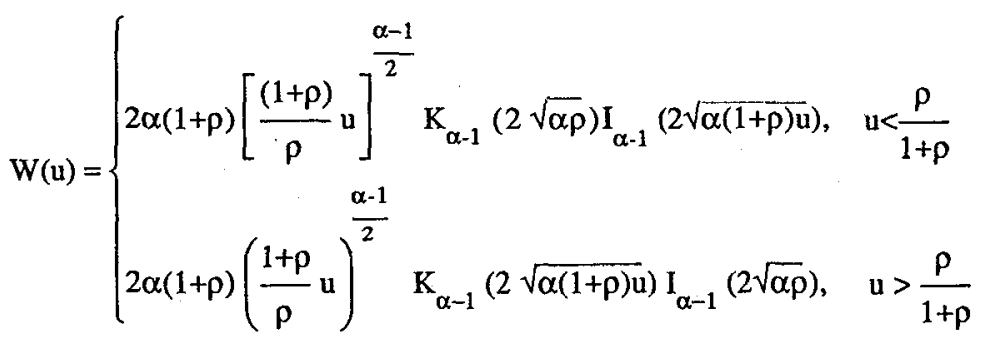

où $u$ est l'intensité normalisée $\mid /<1>, \mathrm{K}_{\alpha}$ et $\mathrm{I}_{\alpha}$ sont les fonctions de Bessel modifiées. Les coefficients $\alpha$ et

$\rho$ sont estimés à partir des expressions (5). Sur les figures 5 el 6 nous avons reporté des histogrammes expérimentaux de $\mid /<1>$ obtenus en faisant varier la distance de propagation dans la turbulence ainsi que la fréquence d'émission de la source acoustique. Les résultats présentés correspondent aux différentes régions du diagramme $\phi-\Lambda$ (Fig. 3). Les comparaisons effectuées avec les quatre distributions définies ci-dessus, mettent en évidence le bon accord avec la loi gamma généralisée quel que soit le niveau des fluctuations. Des résultats similaires ont été obtenus par Ewart et al. /18/ pour des mesures dans l'océan. Pour les deux régimes limites, les distributions expérimentales tendent vers les lois classiques : log-normale lorsque $\beta_{0} \ll 1(f=23.5 \mathrm{kHz} ; x=0.5 \mathrm{~m})$ et exponentielle lorsque $\beta_{\mathrm{o}} \gg 1(f=75 \mathrm{kHz}, x=1.5 \mathrm{~m})$. En ce qui concerne la distribution $1-K$, on observe une surestimation très marquée du maximum de la densité de probabilité, et, en dehors des cas de fortes fluctuations, cette loi de distribution ne permet pas une estimation correcte.

\section{5 - CONCLUSION}

La caractérisation des fluctuations d'intensité d'une onde acoustique traversant une turbulence thermique a été réalisée en laboratoire pour des conditions de propagation variées permettant d'aller des très faibles niveaux de fluctuations jusqu'à l'apparition du phénomène de saturation.

Pour la variance normalisée des fluctuations d'intensité, les résultats expérimentaux sont en accord avec les estimations théoriques associées aux différents régimes (solution de Rytov, solutions asymptotiques de r'équation d'évolution du moment d'ordre quatre). 
Pour la loi de probabilité, nos mesures montrent que les fluctuations d'intensité peuvent être décrites par une loi gamma généralisée quel que soit le niveau des fluctuations. Par ailleurs on retrouve la loi log-normale pour les faibles fluctuations et la loi exponentielle dès que le régime de saturation est établi.

\section{REFERENCES}

$11 /$ Tatarski, V.I., I.P.S.T. Keter Press, Jerusalem (1971).

12 / Ishimaru, A., Wave Propagation and scattering in random media, Vol. 1 et 2, Academic Press, New-York (1978).

$13 /$ Prokhorov, A.M. and al., Proceeding of the I.E.E.E. 63 (1975) 790-811.

$14 /$ Strohbehn, J.W., Topics in Applied Physics, 25 (1978) Springer-Verlag.

$15 /$ Uscinski, B.J., J.O.S.A., 2, 2 (1985) 2077-2091.

$16 /$ Gracheva, M.E. and al., Topics in Applied Physics, 25 (1978) Springer-Verlag.

$17 /$ Furutsu, K., Radio Science, 14 (1979) 287-299.

/ 8 / Blanc-Benon, Ph. and Juvé, D., AlAA 87-2727 (1987) 11th Aeroacoustics Conf., Palo Alto, California.

/9/ Blanc-Benon, Ph., Thèse Docteur ès Sciences, Lyon I, 87-49, (1987).

$110 /$ Andrews, L.C., Philipps, R.L. and Shivamoggi, B.K., Appiied Optics, 27,11 (1988) 2150-2156.

$/ 11 /$ Uscinski; B.J., Macskill, C. and Ewart, T.E., J. Acous. Soc. Am., 74, 5 (1983) 1474-1483.

$/ 12 /$ Gochelashvily, K.S. and Shishov, V.I., Sov. Phys., JETP 39, 4 (1974) 605-609.

/1 3/Parry, G., Optica Acta, 28.5 (1981) 715-728.

114 / Flatte, S.M. and al., Sound Transmission through a Fluctuating Ocean (1979) Cambridge UP., New-York. /15/Strohbehn, J.W., Wang, T. and Speck, J.P., Radio Sciences, 10 1 (1975) 59-70.

116/Dashen, R., J. Math. Phys., 20, 5 (1979) 894-920.

$117 /$ Ewart, T.E., Macskill, C. and Uscinski, B.J., J.Acous.Soc.Am., Z4, 5 (1983) 1484-1499.

/18/Ewart, T.E. and Percival, D.B., J.Acous.Soc.Am., 8Q, 6 (1986) 1745-1753.

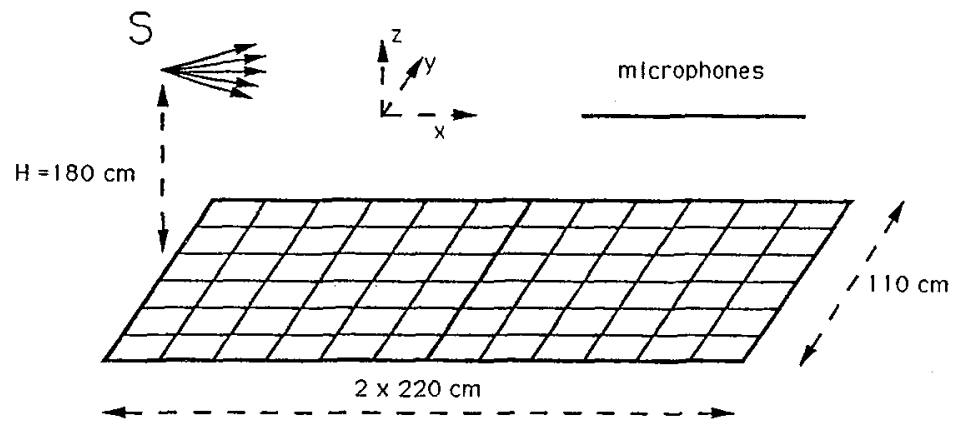

Fig. 1 : Schéma de l'installation expérimentale 


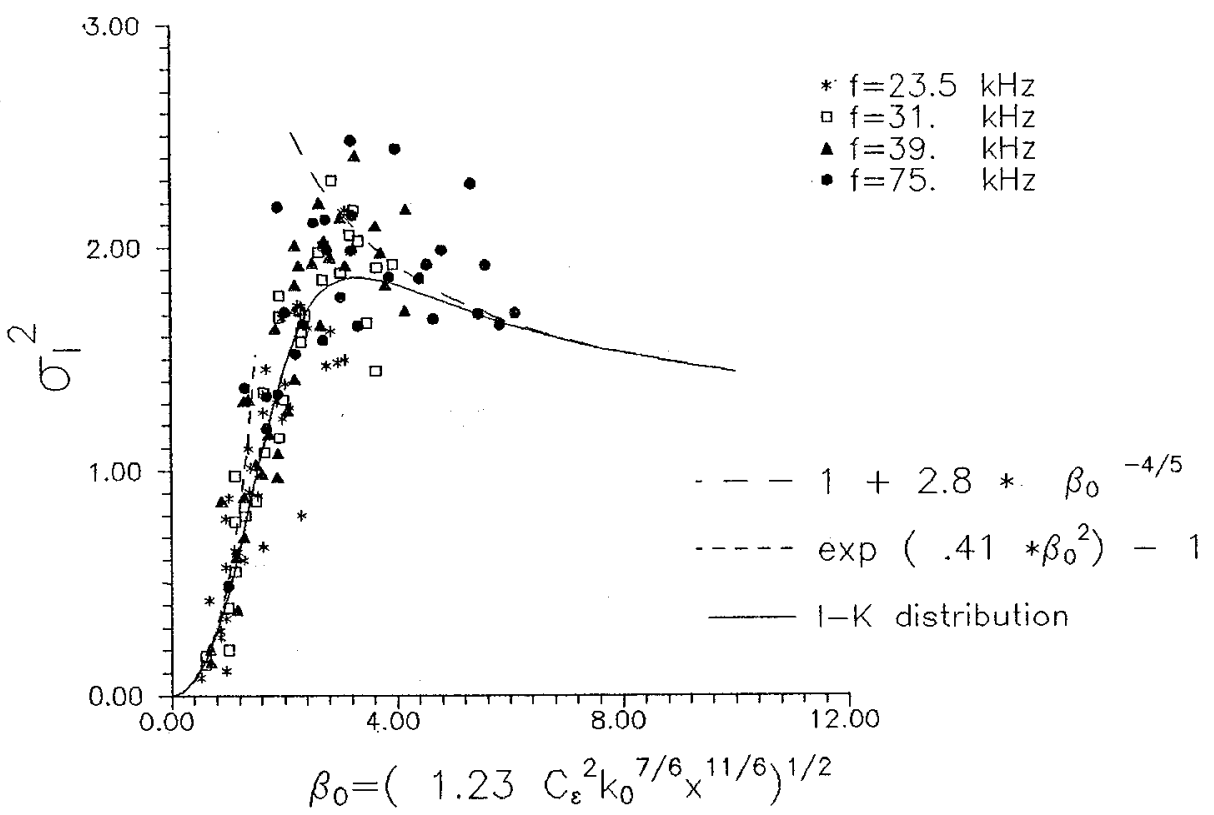

Fig. 2 : Evolution de la variance des fluctuations d'intensité en fonction de la solution de Rytov

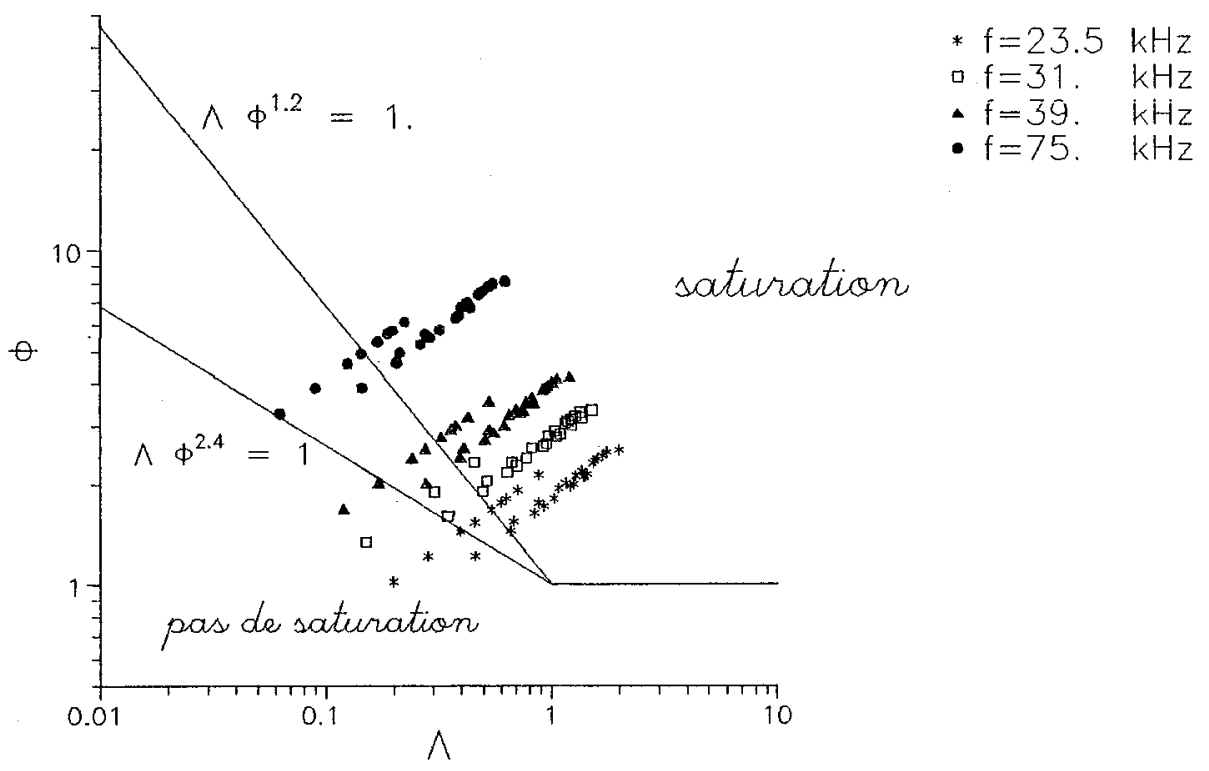

Fig. 3 : Représentation des points expérimentaux dans le diagramme $\phi-\Lambda$ 

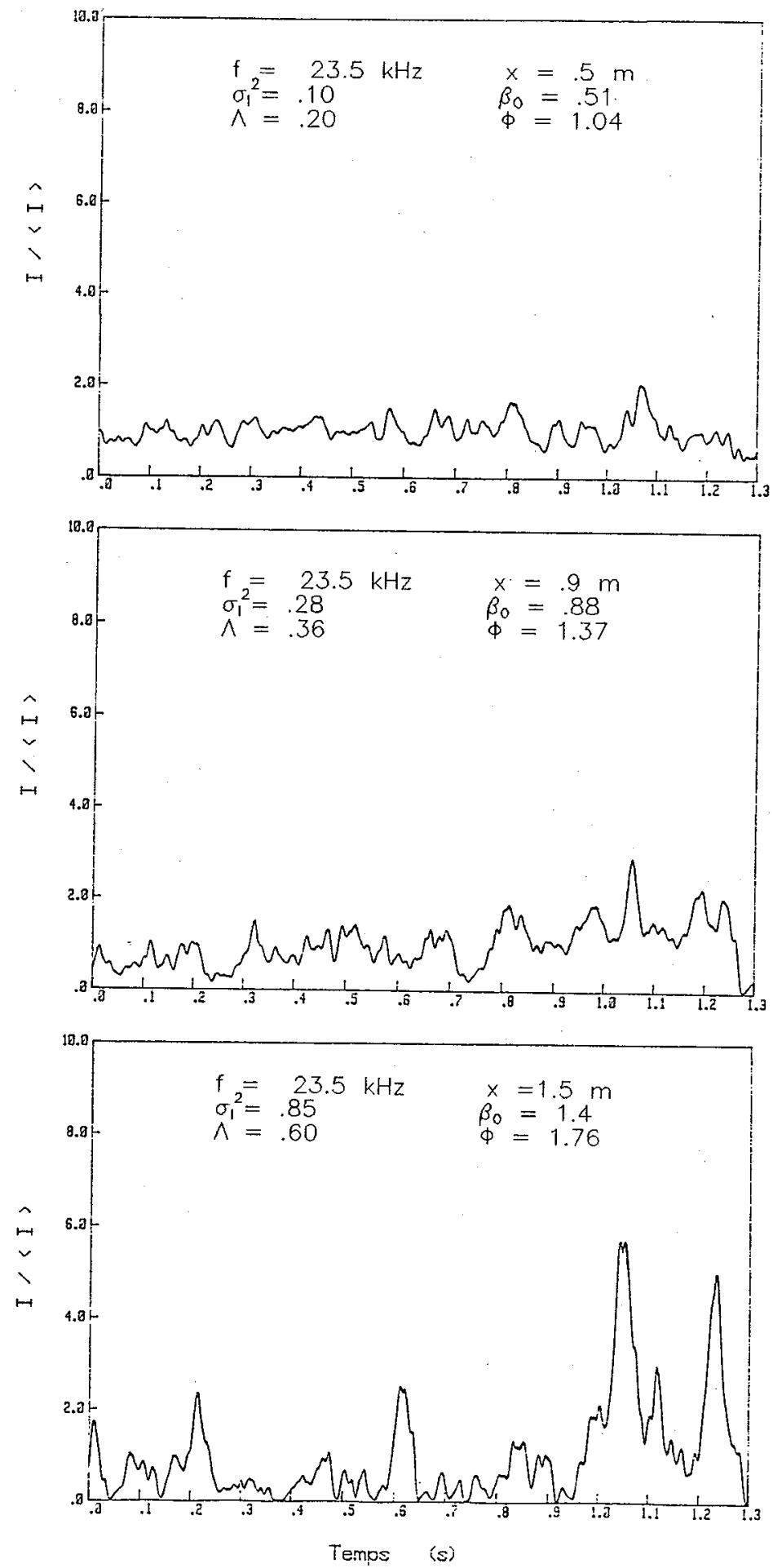

Fig. 4 : Evolution temporelle des fluctuations d'intensité pour differentes distances de propagation 

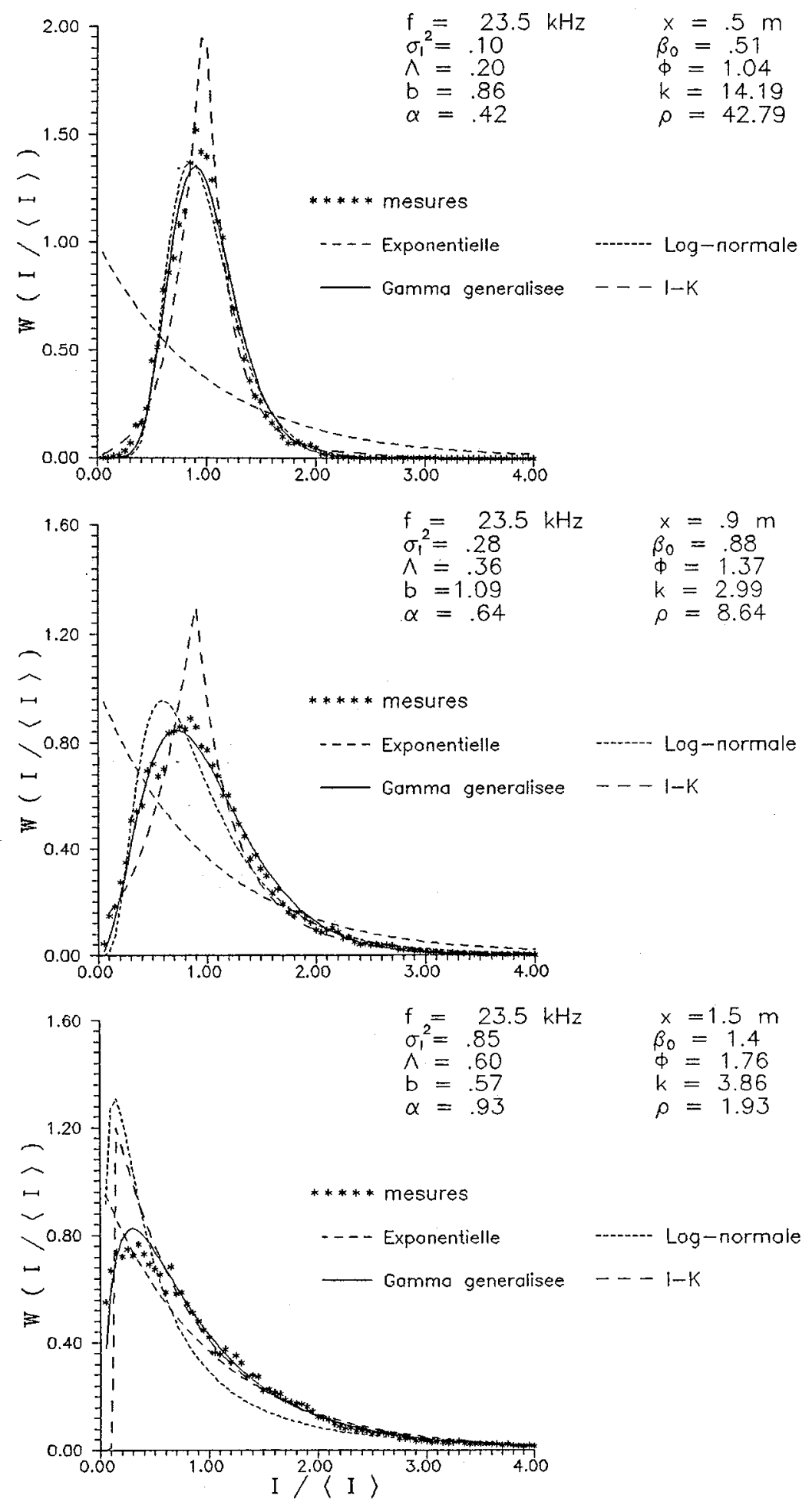

Fig. 5 : Evolution des densités de probabilité $W(I /<l>)$ 

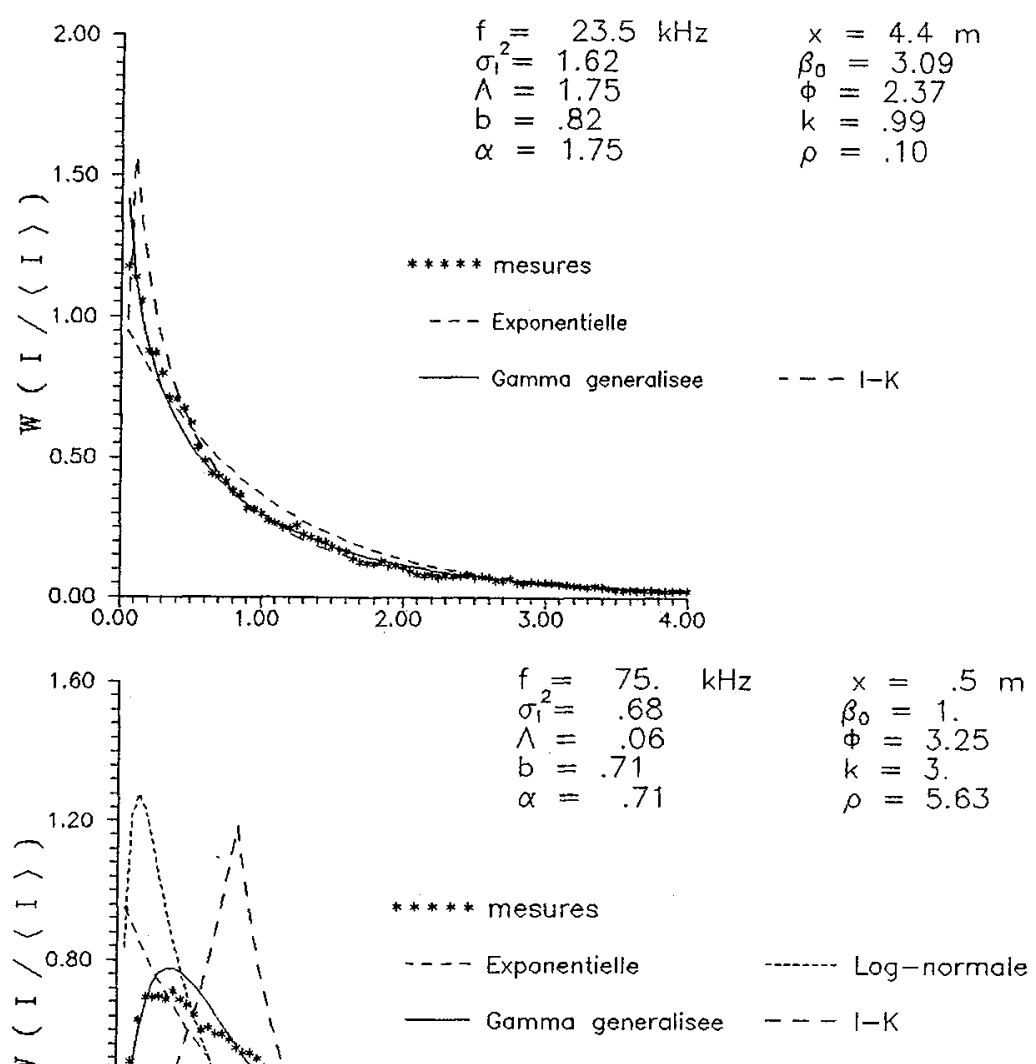

0.
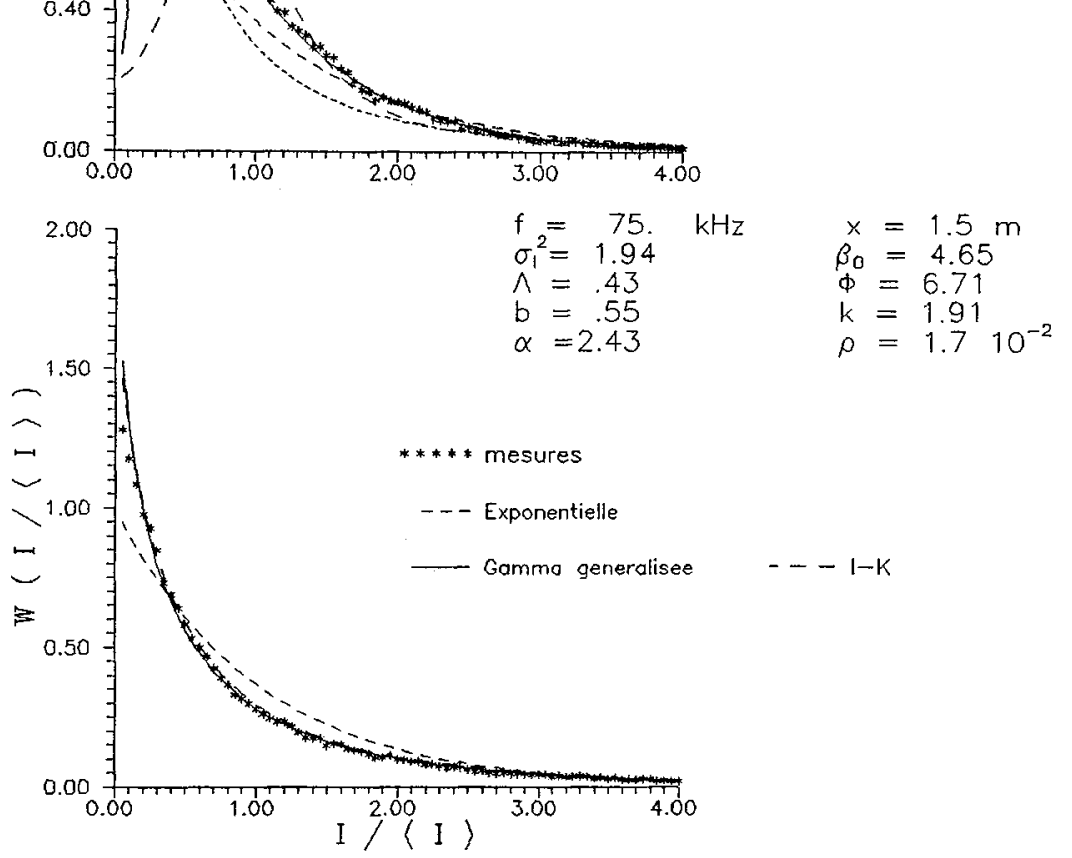

Fig. 6 : Evolution des densités de probabilité $W(1 /<\mid>)$ 